\title{
Inhalational botulism
}

INSERM

\section{Source}

INSERM. (1999). Orphanet: an online rare disease and orphan drug data base.

Inhalational botulism. ORPHA:254504

Inhalational botulism is a man-made form of botulism (see this term), a rare acquired neuromuscular junction disease with descending flaccid paralysis caused by botulinum neurotoxins (BoNTs). 